\title{
Radiation oncology and its Therapeutics
}

\author{
Abhimanyu Mohanta* \\ Department of Zoology, India.
}

\begin{abstract}
The patients suffering from cancer can be undergone with the radiotherapy to kill the cancer cells and this can give the person a great hope to live. Radiation oncology is a medical speciality that involves the controlled use of radiation to treat cancer either for cure, or to reduce pain cause by cancer. Therapeutics refers to the use of drugs and its method of administration for the treatment of various diseases.
\end{abstract}

Keywords: Radiation oncology, Therapeutics, Radiotherapy, Pain, Cancer

Accepted on July 26, 2020

\section{Introduction}

Radiation is a process of killing cancer cells with high doses of radiation and let the tumor cells shrink in the area, therapy used for this process is called Radiation Therapy. Radiation therapy or radiotherapy are often abbreviated as RT, RTx, or XRT, This Radiotherapy is used by ionizing it through radiation this can be generally used as a part of cancer treatment to control or kill malignant cells and normally delivered by a linear accelerator [1].

Medical therapeutics is a branch of medicine that deals with the specific target of the disease and it helps in healing. Radiation Oncologists are the doctors or physicians specifically appointed for the treatment of cancer patients [2-3]. They are highly trained medical professionals advises to deliver the safe and effective radiation therapy with new advanced technologies to treat cancer patients with as few side effects as early as possible [4]. Radiation therapy is a part of treatment in around $40 \%$ of all patients cured of cancer. Across the world the cancer patients with radiotherapy they can highly developed with benefits upon $60 \%$ of people are living happily with their families. About 1 in 2 patients with cancer are seeing benefits with radiation therapy at some point during their illness [5].

Oncology and Therapeutics that conveys the present situation of the clinical and experimental examination in several types of oncology related radiations and some related to the blood referred most commonly as hematology, especially these experimental therapeutics are kept inside for the field of immunotherapy, radiotherapy and chemotherapy [6].

Radiation may be due to harmful rays and this can be treated with radiotherapy here, the success rate of radiation therapy is some cases when patients are treated with modern externalbeam radiation therapy, the overall cure rate was $93.3 \%$ with a metastasis-free survival rate at 5 years of $96.9 \%$. The most common side effects for this radiotherapy are fatigue means feeling tired very early and skin changes can be most seen and other side effects mostly include are related to the place where it is being treated, such as hair loss and mouth problems when radiation treatment is given to this area. Usually this therapy makes the people to feel sick, nausea, in some cases vomiting if in case the treatment area is near the stomach or the brain [6]. This radiation therapy does not kill all the cancer cells they will regrow from the cells that hide, and it may lead to the next stages of the radiotherapy. This cannot be seen upon any type of scanning and blood tests. In some cases when it is given in high doses, the radiation therapy kills cancer cells or then slows down their growth by damaging their DNA [7].

Radiation therapy can be applied to huge number of cancer patients safely with wide range of cancers and may be used alone or in conjunction with surgery, chemotherapy, and other treatments. It is completely non-invasive and accessed through out-patient clinics [8]. Radiation therapy is a highly a costeffective cancer treatment. Radiation oncology is constantly improving day by day. Recent new technologies can benefit some cancer patients which resulting in higher cure rates, fewer side effects, shorter treatments, and improved quality of life [9].

\section{Conclusion}

Oncology patients need the best way of cancer treatment with good benefits and from the use of new medical therapeutic inventions from our world-leading, and with the high quality, safety, and effective radiation oncology services.

\section{References}

1. White N. Principles and practice of radiation therapy. Radiography. 2009;15(4):357-58.

2. Bergenmar M, Johansson H, Sharp L. Patients' perception of information after completion of adjuvant radiotherapy for breast cancer. Eur J Oncol Nurs. 2014;18(3):305-9.

3. Simon MA, Ragas DM, Nonzee NJ et al. Perceptions of patient-provider communication in breast and cervical cancer-related care: a qualitative study of low-income English-and Spanish-speaking women. J Commun Health. 2013;38(4):707-15.

4. Taylor C. Patient satisfaction with information on late effects: Claire Taylor explores what patients who have undergone radiotherapy for pelvic cancers want to know. Cancer Nurs Pract. 2014;13(1):16-22.

5. D'Alimonte L, Hahn S, Makhani L et al. The informational needs of prostate cancer patients treated with radical prostatectomy regarding adjuvant or salvage radiotherapy: determinants of community health pilot project. J Med Imaging Radiat Sci. 2011;42(4):198-209. 
6. Hetz SP, Tomasone. Supportive care needs of Canadian melanoma patients and survivors. Can Oncol Nurs J. 2012;22(4):248-56.

7. Pedersen B, Koktved DP, Nielsen LL. Living with side effects from cancer treatment-a challenge to target information. Scand J Caring Sci. 2013;27(3):715-23.

8. Lynch T. Telephone helpline supports patients with prostate cancer: Teresa Lynch outlines the role of specialist nurses responding to callers asking for information about testing, treatment, and side effects. Cancer Nurs Prac. 2012;11(3):30-4.
9. Grinyer A. The late effects of mantle field radiotherapy: the information and support needs of women survivors of Hodgkin's disease. Eur J Oncol Nurs. 2010;14(3):183-9.

\section{*Correspondence to:}

Abhimanyu Mohanta

Department of Zoology

India

E-mail: abhimanyu.mohanta@gmail.com 